Die Analyse ergab mir:

0.80 pCt. löslich in Wasser fenthaltend: Kieselsäure, Schwefelsäure, Chlor, Kalk, Kali, Natron.

$5.70 \gg 》$ \alzsäure grösstentheils Ferrioxyd und wenig $\overline{100.00}$

Der in Säuren unlösliche Theil enthält:

64.05 Siliciumdioxyd,

6.54 Ferrioxyd (mit sehr wenig Ferrooxyd),

13.08 Aluminiumoxyd,

1.80 Calciumoxyd.

Magnesia, Kali und Natron, Chlor und Schwefelsäure wurden qualitativ bestimmt.

Cliden, im Februar 1884.

252. C.Schmitt und A. Cobenz1: Ueber die Zusammensetzung der im käuflichen Stärkezucker enthaltenen unvergährbaren Substanz und deren Ermittelung. ${ }^{1}$ )

[Erste Mittheilung.]

(Eingegangen am 14. März; mitgetheilt in der Sitzung von Hrn. A. Pinner.)

In den letzten beiden Jahrzehnten ist über den käuflichen Stärkezucker in wissenschaftlichen Zeitschriften vieles, in der Tagespresse aber - angeregt durch die Arbeiten, welche die gesundheitsschädliche Wirkung desselben darthun sollten -- noch viel mehr geschrieben worden, was sich bei näherer Betrachtung als unwahrscheinlich, jedenfalls aber als auf falschen Voraussetzungen beruhend, herausstellen musste.

A. Schmitz sowohl als J. Nessler, Letzterer veranlasst durch die Versuche des Ersteren, haben sich für die gesundheitsschädliche Wirkung des känflichen Stärkezuckers bezw. des damit gallisirten Weines ausgesprochen. Beide schreiben diese Wirkung direct oder indirect den in dem Zucker enthaltenen unvergährbaren Substanzen zu. v. Mering kommt dagegen in seiner Abhandlung: "Enthält der Kartoffelzucker gesundheitsschädliche Stoffe? «, zu dem Resultate: "Die unvergährbaren Bestandtheile des Kartoffelzuckers, welcher aus Stärke-

1) Dic Mittheilung der HHrn. Schmitt und Cobenzl ist ihrer Länge wegen der Publikations-Commission zur Beurtheilung unterbreitet und dadurch der unveränderte Abdruck derselben verzögert worden. Unter dem auf Seite 599 bereits erwähnten Gallisin ist die in diesem Anfsatze näher charakterisirte Substanz zu verstehen.

Die Redaktion. 
mehl durch Kochen mit reiner Säure dargestellt wird, erzeugen nicht nur keine gesundheitsschädlichen Wirkungen, sondern besitzen, da sie zur Gruppe der Kohlenhydrate gehören, einen bedeutenden Nährwerth. «

Durch diese Widersprüche angeregt, hielten wir es um so mehr geboten, dieser Frage näher zu treten, als die grosse volkswirthschaftliche Bedeutung, welche der Herstellung und Verwendung des Stärkezuckers zuzuschreiben ist, von Niemandem bestritten werden kann. Die von uns bei der Bearbeitung des Gegenstandes gewonnenen Resultate werden in allen ihren Einzelheiten, soweit diese die allgemeine, chemische und physiologische Seite der Frage berühren, mit Berücksichtigung aller in Betracht kommenden Umstände, in dem demnächst erscheinenden Jahresbericht unserer Anstalt veröffentlicht werden. Heute wollen wir nur den rein chemischen Theil, soweit er bisher fertig gestellt und für den theoretischen Chemiker Interesse hat, unter möglichster Vermeidung alles Nebensächlichen, behufs Sicherung der Prioritätsrechte zur Kenntniss bringen.

In sämmtlichen Abhandlungen wird von unvergährbarer Substanz oder unvergährbaren Substanzen gesprochen, von der schon Anthon sagte, sie sei kein Gummi mehr, aber auch noch kein Zueker; ihm schliessen sich Mohr und E. Schmitt an, ohne dass sie über deren Eigenschaften mehr sagen, als dass sie der Einwirkung der Hefe widerständen und sich durch fortgesetztes Kochen in Zucker unter Caramelbildung überführen liessen und nicht krystallisirbar seien.

Neubauer war es vorbehalten, in seiner Abhandlung: »Ueber das optische Verhalten rerschiedener Weine and Moste, sowie. über die Erkennung mit Traubenzucker gallisirter Weinek, die unvergährbaren Substanzen weiter zu charakterisiren, und gelang es ihm unter Benutzung der von ihm entdeckten, stark rechtsdrehenden Wirkung eine Methode ausfindig zu machen, mit Hülfe deren es gelingt, die bis dahin zweifelhafte Erkennung der mit Kartoffelzucker befremdeten Weine, nahezu absolut sicher zu stellen. Zur Reindarstellung der Substanzen hat er es aber auch nicht gebracht, denn er schreibt: 》Ich bin mit der weiteren Reinigung dieser Körper, die mit nicht geringen Schwierigkeiten verbunden zu sein scheint, beschäftigt und werde darüber, sofern sie mir überhaupt gelingen sollte, später berichten. So viel glaube ich aber sehon jetzt annehmen zu dürfen, dass wir es hier weder mit Dextrin, noch mit einer Mischung von Dextrin und Zucker zu thun haben, sondern dass in der That, wie auch schon Anthon angiebt, bei der Umsetzung der Stärke ein Zeitpunkt eintrete, bei welchem die Flüssigkeit eine bedeutende Menge eines Stoffes enthält, der kein Gummi mehr, aber auch noch nicht Zucker geworden ist."

Auch Béchamp seheint nach der Angabe von G. Chancel bereits früher einen Körper im Weine gefunden zu haben, der mit dem von $\mathrm{Neubauer}$ beschriebenen eine Aehnlichkeit zu haben schien, wenn 
nicht gar identisch gewesen ist. $r$. Mering bezeichnet in der erwähnten Abhandlung die unvergährbare Substanz als Dextrin. Wir wollen nun sehen, in wie weit die von den erwähnten Autoren gemachten Angaben mit den unserigen übereinstimmen.

Im Anschluss an die oben erwähnte Arbeit von C. Neubauer ward genau, wie dort angegeben, Traubenzucker in Gährung versetzt und nach Beendigung derselben weiter verarbeitet. Ebenso wie Neubauer erhielten auch wir schliesslich zwei syrupdicke Lösungen, die sich aber bald, wie unten gezeigt werden soll, in ihrem Verhalten chemischen Agentien gegenüber so ähnlich, eigentlich gleich verhielten, dass man sie als gleichartiger Natur betrachten konnte.

Schon bei der ersten Wiederholung der erwähnten Angaben, als wir die durch Aetherfällung erhaltene, concentrirte, wässerige Lösung mit 90 procentigem Alkohol schüttelten, erschien es als sehr naheliegend, dass der in Lösung gegangene Theil mit dem ungelöst gebliebenen gleichartig sein dürtte, da dic syrupöse Masse sich einerseits im Wasser in jedem Verhältniss lïst, andererseits aber der 90 procentige Alkohol beim Schütteln mit dem Syrup Wasser aufnimmt und zugleich einen Theil desselben löst. Sagt ja Neubauer selbst, dass sich der mit Alkohol gefällte Theil in 45 procentigem Weingeist vollständig löst, was auch thatsächlich der Fall ist. Unbegreiflich erscheint die Angabe, dass diese erhaltenen Syrupe Fehling'sche Lösung nur noch schwach reduciren sollen, wogegen es sich zeigte, dass das Reduktionsvermögen dieser Syrupe ein so ausgeprägtes ist, dass man den Gehalt solcher Lösungen direkt durch Titriren quantitativ bestimmen kann.

Wir wollen im Folgenden nur beschreiben, welchen Gang wir befolgten und wie wir aus dem Syrup eine chemisch vollkommen bestimmte Verbindung abschieden.

$5 \mathrm{~kg}$ reinen Tranbenzuckers, wie ihn die Firma Remy \& Wahl in Neuwied in den Handel bringt, ward unter Zusatz von Hefe rergähren gelassen. Bei einer Temperatur von $18-20^{\circ} \mathrm{C}$. und einer Concentration von $20 \mathrm{pCt}$. der Lösung war die Gährung nach 5 bis 6 Tagen vollendet. Es wurde filtrirt, die vollkommen helle, fast farblose Flüssigkeit auf dem Wasserbade möglichst stark eingedampft und der erhaltene Syrup noch warm in eine geräumige Flasche gebracht, mit einem grossen Ceberschuss von absolutem Alkohol heftig durchgeschüttelt, wodurch der Syrup sehr zähe ward, sich aber mit dem Alkohol nicht mischte. Letzterer wurde abgegossen, durch neuen, absoluten Alkohol ersetzt und wiederum geschüttelt. Hat man dieses Abgiessen und Schütteln mit neuem Alkohol, welcher zugleich dem Syrup das Wasser und andere Beimengungen, wie noch unvergohrenen Zucker, organische Säuren u. s. w. entzieht, mehrmals wiederholt, so geht der ursprüngliche Syrup schliesslich in eine krümmliche, gelblichgraue Masse über. Diese wird nun in eine grosse Reibschale gebracht 
and mit einem Pistill unter erneutem Zusatz von Alkohol, dem eine gleiche Menge wasserfreien Aethers zugesetzt worden, kräftig durchgeknetet und verrieben. Nach einiger Arbeit zerfällt die ganze Masse zu einem grauen Pulver. Von grosser Wichtigkeit ist, dass das Verfahren des Reibens nicht ausgesetzt werden darf, sowie auch möglichst rasches Arbeiten und thunlichste Vermeidung jeglichen Luftzutrittes sehr zu empfehlen ist, da sonst die Substanz durch Wiederanziehen von Wasser sofort wieder klebrig wird und in einen Syrup zerläuft. Der erhaltene Brei wird möglichst rasch an einer kräftigen Saugpumpe abgesaugt, zunächst mit Alkohol, dann mit Aether nachgewaschen und rasch unter einem Exsiccator mit frischer, concentrirter Schwefelsäure gebracht. Chlorcalcium lässt sich hierbei nicht anwenden, da dasselbe weitaus hygroskopischer ist, als die Substanz selber.

In diesem Stadium ist die Substanz grau und kam zu den meisten Versuchen als genügend rein verwendet werden. Um sie aber rein weiss zu erhalten, wird sie in Wasser gelöst und in ungefähr 40 procentiger Lösung mit viel frisch ausgeglühter, reiner Thierkohle anhaltend gekocht, hierauf wird filtrirt und bis zur starken Syrupdicke auf dem Wasserbad eingedampft. Diese Lösung von lichtweingelber Farbe wird noch warm in ganz dünnem Strahl in einen grossen Ueberschuss eines Gemisches gleicher Theile wasserfreien Alkohols und Aethers im ungefähren Verhältniss von ein Theil Syrup zu mindestens 50 Theilen Gemisch unter kräftigem Schütteln gegossen. Es gelingt auf diese Art, die neue Verbindung als ganz feines, rein weisses Pulver zu erhalten, welches sich schnell zu Boden setzt bei längerem Stehen mit dem Aether. - Alkohol zieht bald das Wasser aus demselben an, zieht und zerfliesst; es muss daher sofort nach der Abscheidung abgegossen und rasch mit einer gleichen Mischung übergossen werden. Hierauf wird die Substanz abgesaugt, wie oben mit Alkohol und Aether gewaschen und unter dem Exsiccator getrocknet.

Die so erhaltene Verbindung, für die wir in der Folge den Namen *Gallisin « vorschlagen, ist vollkommen weiss und hat ungefähr das Aussehen wie Stärkemehl.

Unter dem Mikroskop erweist es sich als vollkommen amorph, ohne irgend welchen charakteristischen Bau. An die Luft gebracht zerfliesst, wie oberr angedeutet, das Gallisin bedeutend rascher, als geglühtes Chlorcaleium in einen dicklichen Syrup, der bald weiter Wasser aufnimmt und dünnflüssig wird. Es ward der Versuch gemacht, ob zwischen dem aufgenommenen Wasser und dem Gallisin ein bestimmtes Gewichtsverhältniss bestehe, und zu diesem $Z_{w e c k e}$ eine gewogene Menge desselben unter eine Glocke neben Wasser gestellt.

$0.5668 \mathrm{~g}$ Substanz nahmen nach einigen Stunden um $0.3602 \mathrm{~g} \mathrm{zu}$, nach ungefähr 12 Stunden im Ganzen um $0.440 \mathrm{~g}$, nach 36 Stunden um $0.579 \mathrm{~g} \mathrm{zu}$. 
Wie ersichtlich, ist also kein bestimmtes Verhältniss zwischen den Gewichten des Gallisins und des aufgenommenen Wassers, da das Gallisin schon nach der ersten Wägung $39 \mathrm{pCt}$. Wasser enthielt, was einer Aufnahme von 12 Molekülen Wasser entspreehen würde, während die letzte Wägung an 20 Moleküle grenzt. Weiter wurde der Versuch als unwesentlich nicht fortgeführt.

Das Gallisin ist in wasserfreiem Aether, Chloroform, Kohlenwasserstoffen unlöslich, äusserst schwer Iöslich in absolutem Alkohol, etwas leichter in Methylalkohol (zum Unterschiede von Glucose, die leicht löslich ist) und Eisessig. Ebenso löst sich Gallisin in einem kochenden Gemisch von gleichen Theilen Eisessig und absolutem Alkohol. Diese Lösungen, die erst durch längeres Kochen erfolgen und durch eine geringe Spur Wasser sehr begünstigt werden, werden auf Zusatz von Aether flockig gefällt. Die flockige Ausscheidung wird nach einigem Stehen pulverig und hart, so dass sie beim Reiben mit einem Glasstab knirscht; es setzt dies voraus, dass keine Spur Wasser in der Lösung war, widrigenfalls entweder der Niederschlag bald klebrig wird, oder die Fällung gleich in syrupösen Tröpfehen gesehieht.

Das Gallisin zeigt in concentrirter, wässriger Lösung, oder wenn es trocken auf Reagenzpapier gelegt wird und an der Luft Wasser anzieht, deutlich saure Reaktion. Die concentrirte, wässerige Lösung zeigt folgendes Verhalten, welches es theils charakterisirt, theils auch von den Zuckerarten, Kohlenhydraten resp. Dextrinen deutlich unterscheidet:

Mit Bleizuckerlösung

»Bleiessig

» Quecksilberchlorid

» salpetersaurem Quecksilber

» Eisenchlorid

》 Jodtinctur

»Chlorcalcium, Chlorbaryum .

keinerlei

Veränderung

auch beim

Erwärmen und

längerem Stehen.

Mit Barythydrat: geringe weisse Fällung, nur in sehr concentrirter Lösung; besonders auf Zusatz von Alkohol.

Mit salpetersaurem Silber: anfangs keine Aenderung, bald aber beim Erwärmen, besonders auf Zusatz von etwas Ammoniak tritt lebhafte Reduktion unter Ausscheidung von metallischem Silber ein.

Uebermangansaures und doppelchromsaures Kali werden lebhaft reducirt. Bei Anwendung des ersteren in schwach alkalischer Lösung tritt beim Schütteln und schwachem Erwärmen sofortige Entfärbung und Abscheidung von Mangansuperoxyd ein. 
Doppelchromsaures Kali in schwach schwefelsaurer Lösung giebt beim Erwärmen bald eine grüne Lösung, wobei zugleich eine lebhafte Gasentwickelung beobachtet werden kann.

Fehling'sche und $\mathrm{Knapp}$ 'sche Lösung werden leicht reducirt (zum Unterschied von Dextrin, welches nicht reducirend wirkt) und ward bei genauerem Verfolg dieser Reaktion folgendes Verhalten gefunden:

1) $0.3348 \mathrm{~g}$ Substanz wurden auf $100 \mathrm{ccm}$ gelöst und mit Fehling'scher Lösung titrirt,

$10 \mathrm{ccm}$ Fehling entsprechen $32.5 \mathrm{ccm}$ obiger Lösung,

$10 \mathrm{ccm} \gg$ 》 $32.6 \mathrm{ccm}$ 》

$10 \mathrm{ccm} \gg \quad$ \ $\quad .05 \mathrm{~g}$ Glucose.

2) $2.1100 \mathrm{~g}$ Substanz wurden auf $200 \mathrm{ccm}$ gelöst, $10 \mathrm{~cm}$ Fehling entsprechen $10.5 \mathrm{~cm}$ dieser Lösung, $10 \mathrm{cem} \gg 10.3 \mathrm{~cm} \gg$ 》

Die Fehling'sche Lösung wird hierbei ungefähr in dem Maasse wie von Milchzucker reducirt.

3) $0.4832 \mathrm{~g}$ Substanz auf $100 \mathrm{ccm}$ gelöst und mit $\mathrm{Knapp}$ 'scher Lösung titrirt, $20 \mathrm{~cm} \mathrm{Knapp} \mathrm{entsprechen} 22.9 \mathrm{ccm}$ dieser Lösung, $20 \mathrm{~cm} D \quad 0.05 \mathrm{~g}$ Glucose.

Die Lösung warde bei dieser Titrirung cubikcentimeterweise zugesetzt. Beobachtet wurde, dass die Knapp'sche Lösung ziemlich schnell und leichter als die Fehling'sche reducirt wird.

In diesen Versuchen also entsprechen demgemäss:

\begin{tabular}{lll}
$0.05 \mathrm{~g}$ Glucose & 1) & $0.10898 \mathrm{~g}$ Gallisin \\
& 2) $0.10972 \mathrm{~g}$ 》 \\
& 3) & $0.110653 \mathrm{~g}$ \\
\hline
\end{tabular}

Im Mittel: $0.109784 \mathrm{~g}$ Gallisin.

Concentrirte Lösungen von Gallisin verhindern die Fällung von Eisensalzen mit Ammoniak oder Alkalihydraten vollständig auch beim längeren Kochen, indem dunkelbraunschwarze Lösungen resultiren, die lebhaft dem Aussehen und dem Geruche nach an Lösungen von Zucker in kochenden Alkalihydratlösungen erinnern. Mit Essigsäureanhydrid wird ein Acetylderivat erhalten.

Bei der Behandlung mit verdünnten Mineralsäuren oder Oxalsäure im Wasserbade geht das Gallisin in Traubenzucker über. Gallisin mit neuer frischer Hefe in wässriger Lösung versetzt, gährt nicht ebenso mit Käseferment; bleibt jedoch Gallisin in verdünnter, rein wässriger Lösung stehen, so zersetzt es sich bald unter lebhafter Schimmelpilzbildung. 
Gallisin giebt schon unter $100^{\circ}$ Wasser und Kohlensäure unter lebhaftem Aufschäumen ab. Der Geschmack des Gallisins ist zuerst ein sehr schwach süsslicher, später fade.

Zur Analyse wurde wiederholt gelöstes und wieder gefälltes Produkt genommen, welches ganz rein, weiss und fein pulverig aussah.

Bei allen vorbereitenden Operationen muss äusserste Vorsicht gebraucht werden, dass die Substanz möglichst wenig mit Luft in Berührung komnt, da sie sonst sofort Wasser anzieht, wie dies auch thatsächlich bei der ersten Analyse geschehen ist. Wasser.

I. $0.3078 \mathrm{~g}$ Callisin gaben $0.4874 \mathrm{~g}$ Kohlensäure und $0.2088 \mathrm{~g}$

II. $0.2632 \mathrm{~g}$ (iallisin gaben $0.4245 \mathrm{~g}$ Kohlensäure und $0.1740 \mathrm{~g}$ Wasser.

III. $0.2532 \mathrm{~g}$ Gallisin gaben $0.4045 \mathrm{~g}$ Kohlensäure und $0.1638 \mathrm{~g}$ Wasser.

\begin{tabular}{|c|c|c|c|c|}
\hline Berechnet & & refund & & Im Mittel \\
\hline für $\mathrm{C}_{12} \mathrm{H}_{24} \mathrm{O}_{10}$ & I. & II. & III. & \\
\hline 43.90 & 43.18 & 43.98 & 43.57 & $43.58 \mathrm{pCt}$. \\
\hline 7.32 & 7.54 & 7.35 & 7.19 & 7.36 \\
\hline
\end{tabular}

Diese Formel, welche nach den analytischen Befunden unter Beibehaltung von $\mathrm{C}_{12}$ als die einzig mögliche erscheint, wird noch durch eine Reihe von Abkömmlingen des Gallisins bekräftigt.

\section{Optisches Verbalten.}

Hierüber haben schon Neubauer und Andere Beobachtungen gemacht, die aber selbstverständlicher Weise der Wahrheit nicht entsprechen können, da sie mit Lösungen operirten, deren Gehalt nach damaligen Umständen nicht genau bestimmt werden konnte.

Leider gelang es auch uns bisher nicht sichere Daten aufzufinden, die die Möglichkeit geben würden, das specifische Drehungsvermögen des Gallisins definitiv festzustellen. Farblose Lösungen von mehr als 54 pCt. Gehalt herzustellen, um sie zur Polarisation zu verwenden, ist nicht gelungen, da die Durchsichtigkeit der Flüssigkeit zu gering wird, nur um, sei es Interferenz, sei es Halbschatten, Beobachtungen zu machen. Letztere Methode mit dem neuester Zeit von Landolt angegebenen und in der Werkstätte von Schmidt und Haensch in Berlin ausgeführten Apparat erlaubte schärfere Einstellung und wurde auch bei unseren Versuchen angewandt. Die Beobachtungen wurden derart angestellt, dass jedesmal die Graddifferenz der Einstellung zwischen einer mit Gallisinlösung und einer mit gleichgefärbtem Wasser gefüllten Röhre gleicher Länge abgelesen wurde. Die Lichtquelle waren leuchtende Gasflammen, also weisses Licht. Mittlere Tempe- 
ratur $20^{\circ} \mathrm{C}$. Andere Lösungsmittel als Wasser konnten leider wegen der sonstigen Unlöslichkeit des Gallisins nicht angewendet werden.

Folgende Versuche wurden ausgeführt:

I. $54.5808 \mathrm{~g}$ Gallisin zu $100 \mathrm{ccm}$ Lösung gebracht gaben eine Drebung von $84.40^{\circ}$ nach Rechts als Mittel von 6 Ablesungen. Rohrlänge $2 \mathrm{dm}$.

II. $27.2904 \mathrm{~g}$ Gallisin zu $100 \mathrm{ccm}$ Lösung gebracht gaben eine Drehung von $43.73^{0}$ nach Rechts als Mittel von 5 Ablesungen. Rohrlänge $2 \mathrm{dm}$.

III. $10.6000 \mathrm{~g}$ Gallisin zu $100 \mathrm{ecm}$ gelöst gaben eine Drehung von $19.20^{\circ}$ im Mittel von 4 Beobachtungen. Rohrlänge $2.2 \mathrm{dm}$.

IV. $7.7540 \mathrm{~g}$ Gallisin zu $100 \mathrm{ccm}$ gelöst gaben eine Drehung von $25.67^{\circ}$ im Mittel von 5 Ablesungen. Rohrlänge $4 \mathrm{dm}$.

Nimmt man die Formel $\alpha_{\mathrm{j}}=\frac{\alpha}{\mathrm{p} . \mathrm{l}}$, wo $\alpha=$ Ablenkung, $\mathrm{p}=$ Gehalt des Cubikcentimeters der Lösung an Grammen Substanz und $\mathrm{I}=$ Rohrlänge in Decimetern, zur Berechnung, so erhält man für $\alpha_{\mathfrak{j}}$ folgende Zahlen:

$$
\begin{array}{r}
\text { I. } \alpha_{\mathrm{j}}=77.32^{\circ} \\
\text { II. } »=80.10^{\circ} \\
\text { III. } "=82.33^{\circ} \\
\text { IV. } »=82.76^{\circ} .
\end{array}
$$

Es scheint also demgemäss das Rotationsvermögen für das Gallisin, wie es aus bisherigen Versuchen, die noch weiter unter Berücksichtigung des specifischen Gewichtes der Lösung und verschiedener Lichtquellen fortgesetzt werden, hervorgeht, bei abnehmender Concentration zu steigen.

Gallisin-Baryum. Wird eine reine, concentrirte, wässerige Lösung des Gallisins mit einer alkoholischen Barytlösung im Ueberschuss von Alkohol versetzt, so entsteht ein flockigweisser Niederschlag, der sich leicht absetzt. Derselbe ward abfiltrirt; durch Waschen mit Alkohol von überschüssigem Baryt befreit und hierauf nochmals in wenig Wasser gelöst und die schwache, trübe Lösung filtrirt, wobei etwas kohlensaures Baryt zurückbleibt und sofort mit viel Alkohol versetzt.

Diese zweite Ausscheidung ist reiner, mehr weiss, als die erste. - Sie wurde abfiltrirt, sorgfïltig mit Alkohol, zuletzt mit Aether gewaschen und unter dem Exsiccator getrocknet.

Vollkommen rein ist diese Verbindung kaum zu erhalten, da sie sich sehr bald unter Gelbfürbung zersetzt, sehr schnell Kohlensüure anziebt und nicht leicht eine constante Zusammensetzung besitzt. In Wasser ist sie sehr leicht löslich. Diese Lösung reagirt deutlich alkalisch, trübt sich sehr bald unter Abscheidung von kohlensaurem Baryt 
und reducirt Fehling'sche und $\mathrm{K} n$ app'sche Lösung. Bei einem analytischen Versuch zeigte sich bald, dass die eben dargestellte Verbindung Wasser gebunden hält, das aber wegen Zersetzung, durch Trocknen bei höherer 'Temperatur nicht bestimmt, hingegen durch (Kewichtsabnahme beim Stehen über Schwefelsäure bestimmt werden kann. Zu diesem $Z$ weck wurde die frisch dargestellte Verbindung, nachdem sie mit Aether gewaschen, über Schwefelsäure gestellt, sofort nach dem Verdunsten des Aethers gewogen und dann bis zum constanten Gewicht über Schwefelsäure stehen gelassen.

I. $0.4030 \mathrm{~g}$ Substanz verloren $0.0414 \mathrm{~g}$ Wasser.

II. $0.2591 * * 0.0271 \%$ *

$$
\begin{array}{cccc}
\multicolumn{2}{c}{\text { Berechnet }} & \multicolumn{2}{c}{\text { Gefunden. }} \\
\text { für } \mathrm{C}_{12} \mathrm{H}_{22} \mathrm{BaO}_{10}+3 \mathrm{H}_{2} \mathrm{O} & \text { I. } & \text { II. } \\
\mathrm{H}_{2} & 10.44 & 10.27 & 10.46 \mathrm{pCt} .
\end{array}
$$

Hexacetylgallisin. Wird Gallisin unter Druck mit dem dreifachen Gewicht Essigsäureanhydrid bei 130--140 2-3 Stunden hindurch behandelt, so erfolgt vollständige Lösung. Die erhaltene bräunliche Masse wird auf Zusatz von Wasser flockig gefällt, nicht aber durch Alkohol. Man kann daher leicht durch Behandeln mit Alkohol das entstandene Produkt von dem nicht angegriffenen Gallisin trennen. Die alkoholische Lösung wird zunächst auf dem Wasserbad, um den Ueberschuss an Anhydrid zu verjagen, eingedampft, wieder mit Alkohol aufgenommen, die Lösung mit Thierkohle entfärbt und unter dem Exiccator verdunsten lassen. Es blieb eine farblose, glasartige Masse zurück, die zerrieben zur Analyse verwendet wurde.

I. $0.1996 \mathrm{~g}$ Substanz gaben $0.3643 \mathrm{~g}$ Kohlensäure und $0.1253 \mathrm{~g}$ Wasser.

II. $0.5452 \mathrm{~g}$ Substanz gaben $0.9944 \mathrm{~g}$ Kohlensäure.

III. $0.2423 \mathrm{~g}$ Substanz gaben $0.4396 \mathrm{~g}$ Kohlensäure und $0.1523 \mathrm{~g}$ Wasser.

$$
\begin{array}{cccccc}
\multicolumn{2}{c}{\text { Berechnet }} & \multicolumn{3}{c}{\text { Gefunden }} \\
\text { für } \mathrm{C}_{12} \mathrm{H}_{18} \mathrm{O}_{4}\left(\mathrm{C}_{1} \mathrm{H}_{3} \mathrm{O}_{2 / 6}\right. & \multicolumn{1}{c}{\text { I. }} & \multicolumn{1}{c}{\text { II. }} & \text { III } & \text { Im Mittel. } \\
\mathrm{C} & 49.65 & 49.78 & 49.74 & 49.48 & 49.67 \mathrm{pCt} . \\
\mathrm{H} & 6.21 & 6.97 & - & 6.98 & 6.97 \text { 》 }
\end{array}
$$

Das Hexacetylgallisin ist, wie schon bemerkt, in Wasser unlöslich, leicht löslich in Alkohol, Aether, Chloroform, Schwefelkohlenstoff, Benzol u. s. w.

Wird Gallisin 4-5 Stunden lang auf $170-180^{\circ}$ mit überschüssigem Essigsäureanhydrid erbitzt, so entsteht viel Essigäther neben wenig Acetylderivat und anderen, nicht näher bestimmbaren, braunen, in Alkohol unlöslichen Massen.

Gallisinkalium. Wird eine absolut alkoholische Lösung des Hexacetylgallisins mit einer Lösung von Kalihydrat in absolutem Al- 
kohol versetzt, so entsteht zunächst ein gelblich gefärbter Niederschlag, der sich beim Schütteln wieder löst, auf Zusatz aber von mehr Kalilösung bis zur eben schwach alkalischen Reaktion, wieder herausfällt. Der entstandene Niederschlag ward abgesaugt, mit Alkohol so lange gewaschen, bis dieser keine alkalische Reaktion mehr zeigte, und schliesslich mit Aether ausgewaschen. Unter dem Exiccator getrocknet, zeigt sich die Verbindung als ein schwach gelblich gefärbtes, bedentend weniger, als das Gallisin, hygroskopisches Pulver, das in Wasser leicht löslich ist. Die erhaltene Lösung reagirt deutlich alkalisch.

Bei obiger Umsetzung ist aber zu bemerken, dass trotz der deutlich alkalischen Reaktion der Lösungen, Essigäther in bedeutenden Mengen auftritt. Diese Erscheinung, sowie der Umstand, dass sich, wie die Analyse zeigt, eine Monokaliumverbindung bildet, deutet darauf hin, dass sich zwar bei fünf Acetylgruppen die Reaktion nach der Gleichung

$$
\mathrm{C}_{12} \mathrm{H}_{18} \mathrm{O}_{4}\left\{\begin{array}{l}
\left(\mathrm{CH}_{3} \mathrm{COO}\right)_{5} \\
\mathrm{CH}_{3} \mathrm{COO}
\end{array}+5 \mathrm{KOH}=\mathrm{C}_{12} \mathrm{H}_{18} \mathrm{O}_{4}\left\{\begin{array}{l}
\left(\mathrm{CH}_{5}\right) \\
\mathrm{CH}_{3} \mathrm{COO} \\
+5 \mathrm{CH}_{3} \mathrm{COOK},
\end{array}\right.\right.
$$

bei einer aber nach der Gleichung

$$
\begin{aligned}
\mathrm{C}_{12} \mathrm{H}_{18} \mathrm{O}_{4}\left\{\begin{array}{l}
(\mathrm{OH})_{5} \\
\mathrm{CH}_{3} \mathrm{COO}
\end{array}+\mathrm{C}_{2} \mathrm{H}_{5} \mathrm{OK}=\mathrm{C}_{12} \mathrm{H}_{18} \mathrm{O}_{4}\left\{\begin{array}{l}
(\mathrm{OH})_{5} \\
\mathrm{OK} \\
+\mathrm{CH}_{3} \mathrm{COOC}_{2} \mathrm{H}_{5}
\end{array}\right.\right.
\end{aligned}
$$

vollzieht. Es wäre hier zu bemerken, dass, wenn man Essigsäureanhydrid mit absolut alkoholischer Aetzkalilösung zusammenbringt, der Geruch nach Essigäther auch bei Ueberschuss von Alkali scharf hervortritt, also die Reaktion wenigstens theilweise nach folgender Gleichung verläuft:

$$
\underset{\mathrm{CH}_{3} \mathrm{CO}}{\mathrm{CH}_{3} \mathrm{CO}} \mathrm{O}+\mathrm{C}_{2} \mathrm{H}_{5} \mathrm{OK}=\mathrm{CH}_{3} \mathrm{COOK}+\mathrm{CH}_{3} \mathrm{COOC}_{2} \mathrm{H}_{5} \text {. }
$$

Das Gallisinkalium scheint nach Vorhergehendem und nach der Analyse einen zum Austauseh gegen Basen geeigneteren Wasserstoff zu haben als die anderen, da sonst wahrseheinlich mehrere Atome Kalium eintreten würden. Ausserdem scheint das Kalium nicht an eine Carboxylgruppe gebunden zu sein.

Das Gallisinkalinm schäumt schon bei schwachem Erwärmen unter Abgabe von Wasser und geringen Mengen von Kohlensäure auf.

Bei der Analyse wurden folgende Daten gefunden:

I. $0.5875 \mathrm{~g}$ Substanz gaben $0.1401 \mathrm{~g}$ schwefelsaures Kali.

II. $0.3077 \mathrm{~g}$ Substanz gaben $0.4432 \mathrm{~g}$ Kohlensäure, $0.1574 \mathrm{~g}$ Wasser und $0.0477 \mathrm{~g}$ Rückstand. 
Ber. für $\mathrm{C}_{12} \mathrm{H}_{23} \mathrm{KO}_{10}$

K $\quad 10.66$

C $\quad 39.34$

H 6.28
Gefunden

I. Th.

$\begin{array}{cc}10.69 & 10.80 \mathrm{pCt} . \\ - & 39.28 》 \\ - & 5.96 \gg\end{array}$

Gallisinblei. Wird eine concentrirte wässerige Lösung von Gallisinkalium mit einer alkoholischen Bleizuckerlösung, nebst einem Ueberschuss von Alkohol, versetzt, so fällt ein flockig weisser Niederschlag aus. Derselbe abfiltrirt, mit Alkohol gewaschen und über Schwefelsäure getrocknet, stellt ein wenig hygroskopisches weisses Pulver dar, das in Wasser sehr leicht löslich ist, desgleichen in alkoholischer Bleizuckerlösung. Ein Zusatz von etwas basisch essigsaurem Bleioxyd, welches die ursprüngliche Fällung ebenfalls sehr begünstigt, fällt die Verbindung sofort wieder. Die wässerige Lösung reagirt alkalisch.

Die Analyse ergab folgendes Resultat:

I. $0.2701 \mathrm{~g}$ Substanz gaben $0.2153 \mathrm{~g}$ schwefelsaures Bleioxyd.

II. $0.2562 \mathrm{~g}$ Substanz gaben $0.2068 \mathrm{~g}$ schwefelsaures Bleioxyd.

\begin{tabular}{|c|c|c|}
\hline Berechnet & & \\
\hline für $\mathrm{C}_{12} \mathrm{H}_{22} \mathrm{PbO}_{10}+\mathrm{PbO}$ & I. & II. \\
\hline 54.76 & 54.46 & \\
\hline
\end{tabular}

Uebergang des Gallisins in Traubenzucker.

Schon Neubauer hat die Angabe gemacht und indirekt den Beweis geliefert, dass diese Verbindung einerseits durch die Einwirkung von verdünnten Säuren auf Stärke entsteht, selbst aber durch dieselbe Wirkung in Traubenzucker übergeht.

Zunächst wurden die Versuche mit den durch Eindampfen der vergohrenen Flüssigkeiten erhaltenen syrupdicken Rückständen gemacht. Dieselben, mit demselben Gewicht von Oxalsäure im Oelbade auf 103-104 2-3 Stunden lang erhitzt, gaben nach dem Absättigen der Oxalsäure mit Kalk, Filtriren und Eindampfen einen dicklichen, rein süss schmeckenden Syrup, der nach wenigen Tagen zu einem Krystallbrei von reiner Glucose erstarrte.

Wichtig war es hiernach zu wissen, ob dieser Uebergang ein quantitativer sei. Es wurden zu diesem Behufe $21.1 \mathrm{~g}$ Gallisin zu $1 \mathrm{~L}$ Wasser gelöst, davon $100 \mathrm{ccm}$ zur Titrirung verwendet und mit den anfangs gegebenen 7ahlen übereinstimmende Resultate gefunden. Der Rest von $900 \mathrm{ccm}$, enthaltend $18.99 \mathrm{~g}$ Gallisin, wurde mit $10 \mathrm{~g}$ reiner Oxalsäure verarbeitet und zwei Stunden auf $105^{\circ}$ èrhitzt, hierauf die Lösung mit kohlensaurem Kalk neutralisirt, das Filtrat auf $1000 \mathrm{ccm}$ 
verdünnt, dann $100 \mathrm{ccm}$ auf $500 \mathrm{cem}$ verdünnt und gegen $\mathrm{Fehling-}$ sche Lösung gestellt.

$$
\begin{gathered}
10 \mathrm{ccm} \text { Fehling } \\
\text { entsprechend } 0.05 \text { Glucose }
\end{gathered}\left\{\begin{array}{l}
22.4 \mathrm{ccm} \text { obiger Lösung. } \\
22.5 》 \gg
\end{array}\right.
$$

$10 \mathrm{ccm}$ Fehling entsprechen also 0.085075 Gallisin. Die Umwandlung war daher noch keine vollständige, es ward der Rest von $900 \mathrm{ccm}$, enthaltend $17.091 \mathrm{~g}$ in Arbeit genommenes Gallisin, mit weiteren $10 \mathrm{~g}$ Oxalsäure 4 Stunden lang erhitzt, hierauf neutralisirt und das Filtrat auf $1000 \mathrm{ccm}$ verdünnt. Davon $100 \mathrm{~cm}$ auf $500 \mathrm{ccm}$ gestellt.

$$
10 \mathrm{ccm} \text { Fehling gleich }\left\{\begin{array}{l}
19.1 \mathrm{~cm} . \\
19.2
\end{array}\right.
$$

$10 \mathrm{ccm}$ F e hling entsprechen also $0.065459 \mathrm{~g}$ verarbeiteten Gallisin.

Die Umwandlung war also noch keine vollendete, der Rest von $900 \mathrm{ccm}$, enthaltend 15.382 Gallisin, ward neuerdings mit $10 \mathrm{~g} \mathrm{Oxal-}$ säure 4 Stunden behandelt.

Diesmal färbte sich die Lösung gelb und roch stark nach Caramel. Nach dem Neutralisiren auf $1000 \mathrm{ccm}$ verdünnt. Davon 100 auf $500 \mathrm{ecm}$.

$$
10 \mathrm{ccm} \text { Fehling gleich }\left\{\begin{array}{l}
22.7 \mathrm{ccm} . \\
22.9
\end{array}\right.
$$

$10 \mathrm{ccm}$ Fehling also gleich $0.07014 \mathrm{~g}$ Gallisin. Die Bräunung und speciell das Zurückgehen der reducirenden Kraft deutet entschieden darauf hin, dass ein Zeitpunkt eintritt, wo entweder der schon gebildete Zucker oder das ursprüngliche Gallisin zerstört wird; eine Beobachtung, die schon Anthon gemacht haben muss.

\section{Oxydation des Gallisins mit Salpetersaure.}

$\mathrm{Zu} 400 \mathrm{~g}$ concentrirter Salpetersänre, die sich in einem grossen Kolben befanden, wurde eine auf $150 \mathrm{ccm}$ gebrachte Lösung von $100 \mathrm{~g}$ Gallisin in Wasser gegossen. Fast augenblicklich tritt schon in der Kälte eine äusserst stürmische Reaktion unter Entwickelung von rothen Dämpfen ein, wodurch sich die Flüssigkeit bis zum Kochen erhitzt. Nach Verlauf der heftigen Einwirkung, die länger als $1 / 2$ Stunde anhält, wurde der Kolben schwach erwärmt, so lange als noch eine Gasentwickelung stattfindet, und hicrauf erkalten lassen, von etwas ausgeschiedener Oxalsäure abfiltrirt, die Lösung bis zur schwach alkalischen Reaktion mit concentrirter Kalilauge versetzt und wieder mit Essigsäure stark angesäuert. In die Kälte gestellt, setzen sich bald schöne, weisse, sternförmig gruppirte Nadeln eines sauren Kalisalzes an, die sich bald vermehren. Es wurde die Lösung abgegossen, die Krystalle abgesaugt und mit kaltem Wasser, in dem sie schwer 
löslich sind, mehrmals nachgewaschen und nochmals aus heissem Wasser umkrystallisirt. Auf diese Weise erhält man das Salz als feine, weisse, seideglänzende Nadeln. Die Mutterlauge der ersten Krystallisation setzt noch weitere Krystalle an, ebenso die der zweiten und dritten u. s. w. Wir erhielten so über $20 \mathrm{pCt}$ : des angewandten Gallisins an reinem Kalisalz. Die wässerige Lösung desselben reagirt deutlich sauer.

Analysirt gab dasselbe folgende Zahlen:

I. 0.2845 g Substanz gab $0.1025 \mathrm{~g}$ schwefelsanres Kali.

II. $0.2731 \mathrm{~g} \gg \geqslant 0.1002 \mathrm{~g}$

III. $0.3017 \mathrm{~g} \gg$ 》 $0.3031 \mathrm{~g}$ Kohlensäure, $0.1044 \mathrm{~g}$ Wasser und $0.0754 \mathrm{~g}$ kohlensaures Kali. $\left.{ }^{1}\right)$

IV. $0.3132 \mathrm{~g}$ Substanz gab $0.3074 \mathrm{~g}$ Kohlensäure, $0.1064 \mathrm{~g}$ Wasser nur $0.0803 \mathrm{~g}$ kohlensaures Kali.

\begin{tabular}{|c|c|c|c|c|c|}
\hline Berechnet & \multicolumn{4}{|c|}{ Gefunden } & \\
\hline für $\mathrm{C}_{6} \mathrm{H}_{9} \mathrm{~K} \mathrm{O} \mathrm{O}_{8}$ & I. & II. & III. & IV. & Im Mittel \\
\hline 29.03 & - & - & 29.57 & 29.00 & $29.28 \mathrm{pCt}$ \\
\hline 3.63 & - & - & 3.84 & 3.77 & $3.81 »$ \\
\hline 15.73 & 16.15 & 16.44 & -. & - & $16.29 »$ \\
\hline
\end{tabular}

Aus diesen Analysen geht demgemäss hervor, dass wir es mit einer zweibasischen Säure von der Formel $\mathrm{C}_{6} \mathrm{H}_{10} \mathrm{O}_{8}$ zu thun haben. Es wurde daher versucht ein neutrales Salz darzustellen und dasselbe in der Form eines Silbersalzes dadurch zu erhalten, indem eine wässerige Lösung des Kalisalzes kalt mit einer solchen von salpetersaurem Silberoxyd versetzt wurde. Es entsteht ein flockiger weisser Niederschlag, der beim Erwärmen krystallinisch wird, sich aber bald grau und endlich schwarz unter Zersetzung fürbt.

Eine Analyse des abfiltrirten und an der Luft getrockneten Niederschlages ergab:

$$
\begin{array}{cr}
0.2088 \mathrm{~g} \text { Substanz gab } & 0.1036 \mathrm{~g} \text { Silber. } \\
\text { Ber. für } \mathrm{C}_{6} \mathrm{H}_{8} \mathrm{Agg}_{2} \mathrm{O}_{8} & \text { Gefunden } \\
\mathrm{Ag} \quad 50.95 & 50.83 \text { pCt. }
\end{array}
$$

Es wurde versucht die freie Säure darzustellen und zu diesem Zwecke die Lösung des Kalisalzes mit Bleizuckerlösung versetzt, der entstandene weisse Niederschlag abfiltrirt, mit kaltem Was ser gewaschen und in Wasser suspendirt, in der Kochhitze mit Scbwefelwasserstoff zerlegt. Das Filtrat vom Schwefelblei gab nach dem Eindampfen einen dicken starksauren Syrup, der nicht krystallisirte. Im Wasser and Alkohol ist er leicht löslich, im Aether nicht.

1) Das Salz schäumt beim Erhitzen sehr stark auf, es kann daher die Kaliumbestimmung bei den Verbrennugen auf Genaugkeit keinen Anspruch machen. 
Die freie Säure wurde zur näheren Charakteristik mit Ammoniak versetzt und der entstandene Krystallbrei von Ammonsalz aus einer kleineren Retorte trocken destillirt. Neben Ammoniak, Pyrrol, das an der mit Salzsäure erhạttenen Rothfärbung gleich erkannt werden konnte, und anderen empyrreumatischen Dämpfen ging auch ein in Wasser, Alkohol und Aether lösliches Sublimat über.

Wir haben sonach Grund zur Annahme, dass wir es hier mit Zuckersäure oder einer Isomere derselben zu thun haben, halten uns zu einem definitiven Ausspruch vorerst nicht berechtigt, hoffen aber in Kurzem in der Lage zu sein, in einer weiteren Mittheilung Näheres hierüber berichten zu können.

\section{Verhalten des Gallisins beim Erhitzen.}

Es wurde Gallisin im Wasserstoffstrom in einem Kölbchen, das sich in einem Wasserbade befand, erhitzt. Das entweichende Wasser und die Kohlensäure wurde in Absorptionsapparaten aufgefangen und gewogen. Scbon bei $65^{\circ}$ fand ein sehr lebhaftes Aufschäumen statt, bei $100^{\circ}$ war die Masse zu einem dicken, zähen, bernsteingelben Syrup geschmolzen und als die Apparate an Gewicht nicht mehr zunahmen, ward der Versuch als beendigt angesehen.

Die Wägung der Apparate ergab eine Zunahme von $17.2 \mathrm{pCt}$. Wasser und $13.7 \mathrm{pCt}$. Kohlensäure, was einer Abspaltung von $4 \mathrm{Mol}$. Wasser (Ber. $18.0 \mathrm{pCt}$.) und 1 Mol. Kohlensäure (Ber. $13.4 \mathrm{pCt}$.) entspricht.

Die im Kölbchen zurückgebliebene und nach dem Erkalten glasartig gewordene Masse hat den äusseren Eigenschaften nach das Verhalten des Gallisins und konnte vorläufig nur eine Baryumverbindung nach der beim Gallisinbaryum angegebenen Weise erhalten werden.

Dieselbe, im Aeussern dem Gallisinbaryum ähnlich anssehend, ergab bei der Analyse folgende Zahlen:

I. $0.3145 \mathrm{~g}$ Substanz gab $0.1024 \mathrm{~g}$ schwefelsauren Baryt.

II. $0.2370 \mathrm{~g}$ Substanz gab $0.0766 \mathrm{~g}$ schwefelsauren Baryt.

\begin{tabular}{ccc}
$\stackrel{\text { Berechnet }}{\text { Bür }}\left(\mathrm{C}_{11} \mathrm{H}_{23} \mathrm{O}_{8}\right)_{2} \mathrm{Bat}$ & \multicolumn{2}{c}{ Gefunden } \\
$\mathrm{Ba} \quad 19.49$ & I. & II. \\
Ba & 19.14 & $19.00 \mathrm{pCt}$.
\end{tabular}

Bis jetzt kann aus Vorhergehendem geschlossen werden, dass das Gallisin wohl Wasser und Kohlensäure abgespalten hat, ersteres aber beim Auflösen im Wasser wieder aufgenommen worden ist, da durch die Verbindungszahlen der Baryumverbindung blos auf eine $\mathbf{A b -}$ spaltung von einem Molekül Kohlensäure gedeutet wird. Nähere Aufklärung kann erst durch die eventuelle Isolirung der Verbindung selust erreicht werden. 
Nachweis des Gallisins im gallisirten Wein.

Es war selbstverständlich von hohem Interesse, die Verbindung im gallisirten Wein direkt nachzuweisen und ehemisch zu bestimmen. $\mathrm{Zu}$ diesem $Z_{\text {weck }}$ ward ein gallisirter Wein von bekannter Herkunft und Zusammensetzung dahin verarbeitet. Der Wein (nach den zuverlässigen Angaben des gegenwärtigen Besitzers) wurde aus rheinpfälzischen Trauben im Jahre 1873 unter Zusatz von Stärkezucker bereitet und enthielt unter Anderem nach einer im November vergangenen Jahres ausgeführten Analyse in $100 \mathrm{~cm}$ in Grammen ausgedrückt:

\begin{tabular}{|c|c|c|c|}
\hline & & & \\
\hline Freie Säure $=$ & $\left.\mathrm{H}_{6} \mathrm{O}_{6}\right)$ & & \\
\hline Extrakt & & & \\
\hline Mineralstof & & & 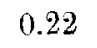 \\
\hline & & & \\
\hline Veinstei & & & 0.1 \\
\hline
\end{tabular}

und zeigte im Wild'schen sowie im Lippich-Landolt'schen Polaristrobometer bei $220 \mathrm{~mm}$ Röhre nach dem Behandeln mit 1/10 Volumen Bleiessig eine Rechtsdrehung von $8.33^{\circ}$.

Es wurde eine grosse Menge desselben in einer Schale auf dem Wasserbade eingedampft und der rothbraune Rückstand von sehr zäher Consistenz mehrmals in der Wärme mit viel gtwöhulichen Methyl alkohol behandelt. Während durch Fällen der wässrigen Lösung des Rückstandes mit Aethylalkohol die Extraktivstoffe mit in den Niederschlag gehen würden, gelingt es auf obige Art eine Lösung zu erhalten, die verhältnissmässig wenig Extraktivstoffe enthält. Dieselbe zunächst mit Thierkohle behandelt, gab auf Zusatz von viel absolutem Aethylalkohol eine weisse, flockige Fällung, die rasch abgesaugt, mit Alkohol gewaschen und dann nochmals in sehr wenig Wasser gelöst wurde. Die erhaltene, fast ganz helle Lösung wird unter heftigem Schütteln in ein kaltes Gemisch gleicher Theile absoluten Alkohols und Aethers gegossen.

Man erhält so eine rein weisse, pulvrige Fälllung, die in jeder Weise die charakteristischen Eigenschaften des Gallisins zeigt.

Fine Analyse daron gab folgende Zahlen: $0.3873 \mathrm{~g}$ gaben $0.6227 \mathrm{~g}$ Kohlensäure und $0.2575 \mathrm{~g}$ Wasser.

$\begin{array}{ccc}\text { Berechnet anf } \mathrm{C}_{12} \mathrm{H}_{24} \mathrm{O}_{10} & \text { Gefinden } \\ \mathrm{C} & 43.90 & 43.85 \mathrm{pCt} . \\ \mathrm{H} & 7.32 & 7.39\end{array}$

Es wurde die Barytverbindung in der schon angegebenen Weise angestellt und auch hier dieselben Eigenschafen gefunden.

Ebenso konnte das auch seine Lnlöslichkeit in Wasser und Leichtlöslichkeit in Alkohol, Aether, Benzol u. s. w. charakterisirte Acetylderivat erhalten und bestimmt werden. 
Zum Schlusse dieser Mittheilung bemerken wir noch, dass nach den bei den physiologischen Versuchen, die sowohl mit Thieren wie mit Mensehen in umfangreicher Weise an unserer physiologisch-chemischen Abtheilung von Hrn. Dr. A. Pfeiffer ansgeführt worden, von einer direkten oder indirekten gesundheitsschädlichen Wirkung des Gallisins oder des damit behafteten Traubenzuckers nicht die Rede sein kann.

Es ist demnach böchlichst zu bedauern, dass mehrfach Leute, welche Stärkezucker zum Gallisiren des Weines verwendet hatten, ohne damit ihre Abnehmer täuschen zu wollen, lediglich deshalb zu schweren Geldstrafen und theilweise Gefängniss verurtheilt wurden, weil sie fahrlässiger Weise gesundheitsschädliche Stoffe verkauft haben sollten.

Wiesbaden, Schmitt's Laboratorium.

\section{W. Spring: Bemerkungen über ein Referat des Herrn Dr. Gabriel.}

(Eingegangen am 22. März.)

In einem im Hefte No. 4 dieser Berichte erschienenen Referate über eine jüngst veröffentlichte Abhandlung des Herrn E. Jannettaz fasst Herr Dr. Gabriel jene Abhandlung so auf, als sei sie eine neue Widerlegung meiner Experimente über die Bildung chemischer Verbindungen durch Druck. Da diese Auffassung eine irrthümliche ist, denn sie schreibt Herrn Jannettaz eine Meinung $z u$, welche er nicht mehr hat, und da in Folge dessen experimentell bewiesene Thatsachen in Miscredit gebracht werden können, so sei es mir gestattet sie zu berichtigen.

Was die Bildung von Verbindungen durch Druck anbelangt, sagt Herr Jannettaz folgendes: "Nous reconnaissons tout d'abord que des combinaisons peuvent se produire entre des corps, même à l'état de poudres sêches, sous l'influence d'une forte pression. . . . . . . Après la publication de la note de M. Spring, nous avons vu que le désaccard était un pur malentendu. M. Spring ne parvient à combiner, dans ces conditions (nämlich wenn man nicht mehr wie einmal das Pulvergemisch comprimirt) qu'une faible partie des éléments; ce n'est qu'après avoir plusieurs fois répété la même opération qu'il arrive à combiner la masse plus ou moins complêtement. "

In seiner Abhandlung hat Herr Jannettaz nur den Zweck, zu beweisen, dass durch eine einzige Pressung nur einige Tausendstel der 